\title{
Prevalence and Antibiotic Susceptibility of Bacterial Pathogens Implicating the Mortality of Cultured Nile Tilapia, Oreochromis niloticus
}

\author{
Hassan, S. E. ${ }^{\sharp 1}$; Abdel-Rahman, M. A. ${ }^{* 1}$; Mansour, E. ${ }^{2}$; Monir, W. ${ }^{1}$ \\ ${ }^{1}$ Botany and Microbiology Department, Faculty of Science, Al-Azhar University, \\ Cairo, Egypt \\ ${ }^{2}$ Bacteriology Department, Animal Health Research Institute, Zagazig Branch, Egypt \\ *Corresponding author: mohamedali@kyudai.jp \\ \#Both authors are contributed equally to this manuscript
}

Received: Dec. 30, 2019; Accepted: Feb.22, 2020 published: 2020 Vol.10 (1):23-43

\begin{abstract}
Nile-Tilapia (Oreochromis niloticus) aquaculture represents one of the most important cultivation species in Egypt. However, Tilapia fish farming is challenged by some problems. Of those, the presence of bacterial pathogens resulting in high fish mortalities and huge economic losses. Thus, the current investigation aimed to isolate, identify, and characterize the pathogenic bacteria from Nile tilapia fish farm in El-Abassa village, Egypt and to investigate their antibiotic susceptibility as a primary step for controlling diseases. 182 bacterial isolates were obtained from one hundred Tilapia fish samples. The microbiological and biochemical analysis of the examined fish indicated the presence of only 5 bacterial genera. Three of them are Gram-negative bacteria (representing $86.26 \%$ of total isolates) including Aeromonas spp. (46.70\%), Pseudomonas spp. (23.08 \%), and Vibrio spp. (16.48 \%). While two genera are Gram-positive bacteria (representing $13.74 \%$ of the total isolates) including Streptococcus spp. $(8.79 \%)$ and Staphylococcus spp. (4.95\%). This indicates that Gramnegative bacteria are the main cause of high fish mortalities in the studied area while Aeromonas hydrophila exhibited the highest prevalence in infected tilapia. Antibiogram test revealed high levels of resistance expressed by all isolates to ampicillin, amoxicillin, and erythromycin. On the other hand, norfloxacin was effective against all isolated bacteria followed by ciprofloxacin; therefore, norfloxacin should be
\end{abstract}


recommended as a supplement in fish fed-diets to control the bacterial infection. Establishing effective control methods for pathogenic isolates would greatly enhance fish production.

Keywords: Fish mortality; Nile tilapia; Oreochromus niloticus, pathogenic bacteria; sensitivity test; Aeromonas spp.

\section{INTRODUCTION}

Tilapia aquaculture is one of the most important aquacultures in fish production in Egypt because of its tolerance to poor water quality and can fed on a wide range of natural food organisms (Shaheen et al., 2013). Amongst them, Nile-tilapia (Oreochromis niloticusis) is of the most important freshwater species for commercial aquaculture because of its high nutritional qualities, fast growth rate and resistance to diseases (Mapenzi and Mmochi 2016). Egypt is the second-largest producer of farmed tilapia next to china (FAO, 2019). Nile tilapia production in Egypt contributes about $65.15 \%$ of Egyptian fish production (GAFRD, 2017). However, under intensive farming, tilapia becomes sensitive to several aquatic pathogens such as bacteria, fungi, parasites, viruses and water molds (Plumb and Hanson, 2010).

Bacteria are the main pathogens of cultured warm water fish involved in great losses to the aquaculture industry elsewhere. Many bacteria are considered to be saprophytic that exists in a commensal association with the host or live free in the environment while others are opportunistic. These bacteria can cause diseases when the immunity of fishes gets decreased by the effect of different stressors (Briede, 2010). Some of the pathogenic bacteria that can cause infection in tilapia fish include Pseudomonas spp., Aeromonas spp., Vibrio spp., Streptococcus spp., Micrococcus spp., Enterococcus spp., Plesiomonas spp., Staphylococcus spp., Moraxellaceae, and Enterobacteriaceae (Zahran et al., 2016). Aeromonas hydrophila, Pseudomonas fluorescens and Vibrio anguillarum were the most predominant bacteria that cause infection in fish farms and contributed to the $O$. niloticus seasonal summer mortalities (Abd El-Kader and Balabel, 2017).

Bacterial infections in fish may occur as bacteremia which means that the bacterial organisms are existent in the blood-stream with no clinical signs or occur as septicemia which implies that bacteria and toxins are indeed present in the circulatory system and always cause disease and clinical signs. Hemorrhage, inflammation, and necrosis are clinical signs associated with septicemia (Briede, 2010). Aeromonas hydrophila, a widely distributed in aquatic environments, is a causative agent of motile 
Aeromonas septicemia (MAS) (Pridgeon et al., 2011). The infected fish showed loss of appetite, loss of equilibrium, scales loss, sluggish swimming at the water surface, exophthalmia, skin erosions and ulcer, fin and tail rot, enlarged abdomen with ascites and vent was prolapsed. Gills might be congested or pale and anemic and covered with excessive mucus. The internal organs may friable and showed a generalized hyperemic appearance (Hassan et al., 2011).

Therefore, the current investigation aimed to isolation and identification of bacterial pathogens from naturally infected Nile tilapia (Oreochromis niloticus) with special reference to the best effective antibiotics for controlling the infection.

\section{MATERIAL AND METHODS}

\section{Naturally infected fish}

A hundred of naturally infected tilapia of different body weights and lengths were randomly collected from El-Abassa Fish Farm, Sharkia Governorate. The fish were transferred alive in tanks containing pond water to the microbiological lab in the Fish Diseases Department, Central Lab for aquaculture Research in Abbassa, Sharkia. All collected samples were subjected to clinical, postmortem and bacteriological examinations as described by Austin and Austin, (2012) and Noga, (2010).

\section{Bacterial isolation}

Under complete aseptic conditions, the specimens of different tissues and organs (skin ulcer, tail, gills, liver, kidneys, and spleen) from diseased fish were inoculated into Tryptic soy broth (Difco) and incubated at $27^{\circ} \mathrm{C}$ for $24 \mathrm{~h}$. Then loopful of broth were streaked onto Tryptic soy agar (TSA) plates and TSA plates with $2 \% \mathrm{NaCl}$ and incubated at $27 \pm 1^{\circ} \mathrm{C}$ for $48 \mathrm{~h}$. For purification purposes, pure colonies were further sub-cultured into Tryptic soy agar. Each type of culture colony was picked up and sub-cultured on a selective diagnostic agar media (Thiosulphate citrate bile salt agar (Oxoid) (TCBS), Aeromonas base agar media supplemented with ampicillin (5 $\mathrm{mg} / \mathrm{L}$ ) and Pseudomonas base agar at $27^{\circ} \mathrm{C}$ for $48 \mathrm{~h}$. Then pure colonies were transferred onto TSA slant for further identification that carried out according to Austin and Austin, (2012) and Quinn et al., (2002).

\section{Bacterial Identification}

The obtained bacterial isolates were subjected to full identification by using colonies characteristics, Gram stain, and biochemical activities as previously described (Austin and Austin, 2012; Quinn et al., 2002). 


\section{Antibiotic sensitivity test}

Sensitivity tests for isolated bacteria to eight types of commercial antibiotic, namely: norfloxacin (NOR), ciprofloxacin (CIP), gentamicin (GN), amoxicillin (AMX), amikacin (AK), rifamycin (RF), erythromycin (E) and ampicillin (AM) were performed on Mueller-Hinton agar (Oxoid) using the disc diffusion method according to the National Committee of Clinical Laboratory Standards (NCCLS) (2003). The diameters of the inhibition zone appearing in the agar plate were measured and interpreted as susceptible (S), intermediate (I) or resistant (R).

\section{RESULTS}

\section{Examination of naturally diseased fish}

\section{Clinical picture}

The clinical picture of the collected naturally infected Nile tilapia (Oreochromis niloticusis) fish showed anorexia, body depigmentation, frayed fins and tail, corneal opacity, exophthalmia, body ulceration, detachment of scales, hemorrhages all over the fish body especially at fins and tails. In some cases, the infected fish showed erythema around the mouth and swelling of the abdomen (Figure 1).

\section{Postmortem picture}

Postmortem examined showed that the infected fishes suffered from congestion in gills, splenomegaly, congested liver with distended gall bladder, enlarged and dark congested kidney and some cases showed a change in liver color (pale or green) and ascetic fluid which was yellowish in color and watery inconsistency (Figure 2). 

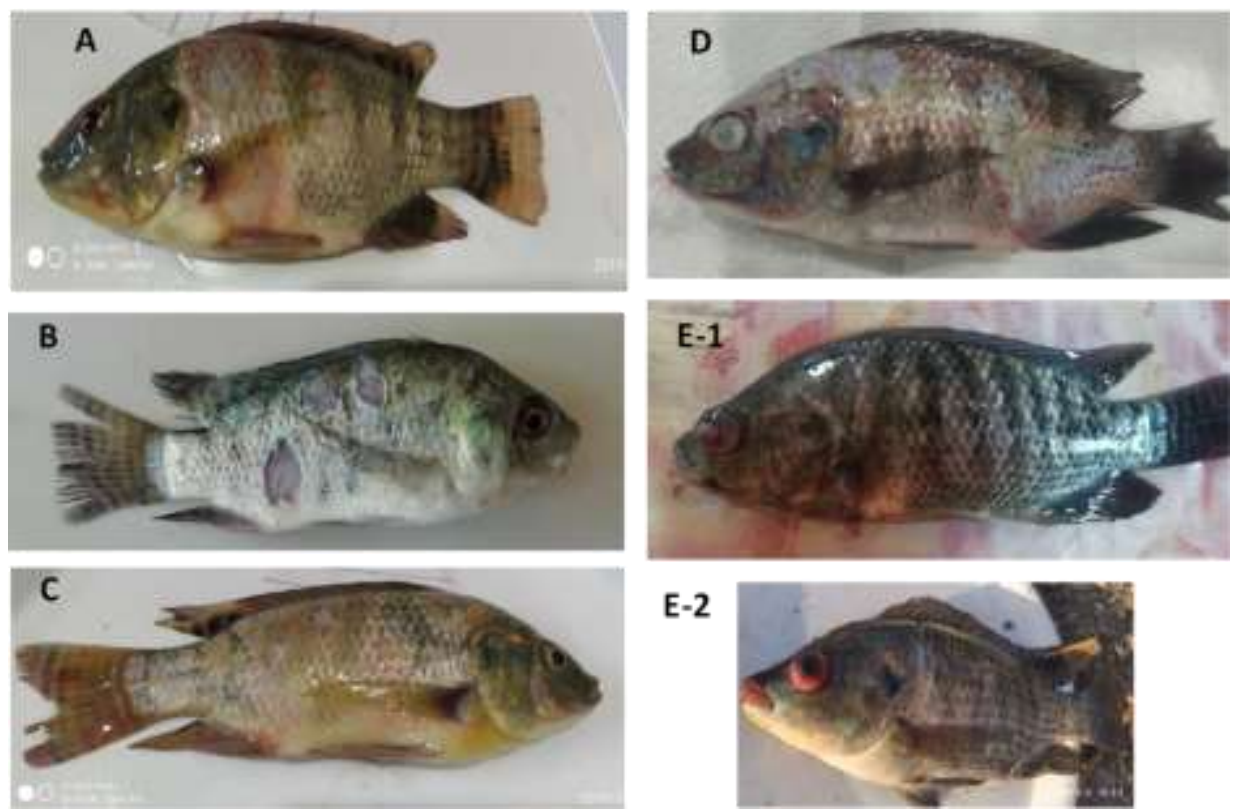

Figure (1): Naturally infected fish showing hemorrhage, scales loss and fin and tail rot (A), skin ulceration (B); Frayed tail and scales loss (C); Corneal opacity and Scales detachment (D) and Exophthalmia (E-1\&2).

Figure (2): A, naturally infected Tilapia showing abdominal swelling, B, Postmortem examination showing enlargement of gall bladder, yellowish liver, empty intestine and congestion in kidney, and $\mathrm{C}$, Congested gills and yellowish ascetic fluid.
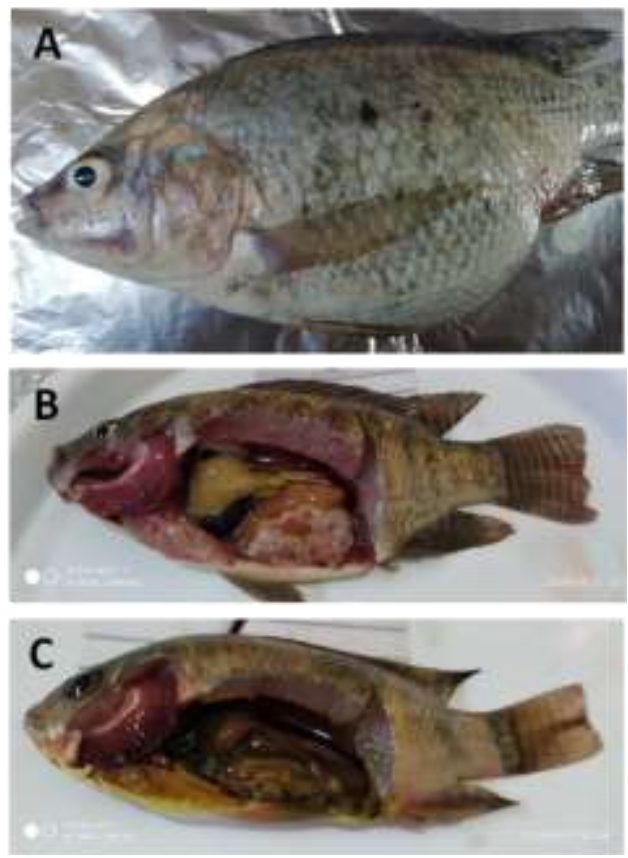


\section{Isolation and identification of pathogenic bacteria}

One hundred and eighty-two bacterial isolates were isolated from the infected fish. These isolates were phenotypically identified following the standard protocol described bacterial isolation and identification. Out of these isolates, 157 isolates $(86.26 \%)$ were Gram-negative bacteria. Those are identified as Aeromonas spp., Pseudomonas spp. and Vibrio spp. Ther other 25 isolates at rate of (13.74 \%) of total isolated strains were Grampositive bacteria that identified as Streptococcus spp. and Staphylococcus spp.

Aeromonas spp. were identified biochemically to A. hydrophila, A. veronei, A. sobria, A. jandae, and A. cavae. All the isolated Aeromonas species were Gram-negative, short rod, motile in semisolid media, positive for oxidase, fermentative, and produce catalase. The data showed in Table (1) demonstrated the full identification scheme of isolated Aeromonas spp.

Pseudomonas spp. were identified biochemically to $P$. fluorescens, $P$. Aeroginosa, and $P$. anguilliseptica. All isolated Pseudomonas spp were Gram-negative, long curved rods, motile and oxidase-positive as indicated in Table (1).

On the other hand, the Vibrio spp. were further identified as V. harveyi, $V$. vulnificus and $V$. alginolyticus. All isolated Vibrio spp. were Gramnegative, curved rods, fermentative, and positive for oxidase, catalase, and methyl red. $V$. harveyi and $V$. alginolyticus produced yellow colonies on TCBS media while $V$. vulnificus produced green colonies. The complete characterization was shown in Table (1).

As shown in Table (2), Streptococcus spp. were cocci, non-motile, oxidase negative, $\mathrm{O} / \mathrm{F}$ fermentative, catalase negative, can grow in media with 3.5 and $6 \% \mathrm{NaCl}$ but not grow in media with $8 \% \mathrm{NaCl}$, hydrolyzed starch, negative for methyl red and Vogaus Proskauer. They cannot utilize citrate as a sole carbon source and produce acid from sucrose, glucose, maltose and fructose but not from inositol, and variable for arabinose, salicin, mannitol and lactose. While Staphylococcus spp. as showed in Table (2), were cocci, non-motile, oxidase negative, oxidase negative, $\mathrm{O} / \mathrm{F}$ oxidative, catalase positive, can grow in media with $3.5,6$ and $8 \% \mathrm{NaCl}$, cannot hydrolyze starch, positive for methyl red and Vogaus Proskauer. They can utilize citrate as a sole carbon source, positive for gelatin liquefaction and produce acid from sucrose, glucose, maltose, mannitol, lactose and fructose but not from arabinose, salicin, and inositol. 


\section{Prevalence of bacterial infections among the examined Nile tilapia fish}

From the result in Figure (3), the prevalence of isolated bacteria was as the following. The highest prevalence rate of the isolated bacteria from naturally infected Nile tilapia fish was Aeromonadaceae (46.70\%) as $A$. hydrophila with prevalence rate (16.48\%), A. veronei (14.84\%), A. sobria (6.59\%), A. jandae $(6.04 \%)$ and A. cavae with prevalence rate $(2.75 \%)$. followed by the Pseudomonadaceae $(23.08 \%)$ as $P$. fluorescen with prevalence rate $23.08 \%, P$. Aeroginosa $(10.44 \%)$, and $P$. anguilliseptica $(8.79 \%)$. While Vibrionacea has prevalence rate $16.48 \%$ as $V$. harveyi at (7.14\%), V. vulnificus at (5.49\%). and V. alginolyticus at (3.85\%). On the other hand, the prevalence of Streptococcus was (8.79) \% and the Staphylococcus have lowest prevalence rate at (4.95\%).

\section{Incidence of isolated bacteria in the organs and tissues}

As shown in Table (3), the most occurrence of the isolated bacteria was from the liver $(37.3 \%)$ followed by the kidney $(25.8 \%)$, spleen $(15.3 \%)$, gills $(10.4 \%)$, tails $(7.14 \%)$, and skin ulcer $(3.84 \%)$.

\section{Sensitivity of the pathogenic isolates to antibiogram}

The sensitivity of the isolated bacterial species, obtained from naturally infected fishes in this study, to different antibiotics was evaluated as indicated in Table (4). As showed, high levels of resistance were expressed by all isolates to ampicillin, amoxicillin, and erythromycin. On the other hand, norfloxacin was drug of choice against all isolated bacteria followed by ciprofloxacin that is effective against most bacterial isolates under study.

\section{DISCUSSION}

Nile tilapia is the main cultured fish species in Egypt. Tilapia fish are susceptible to several bacterial diseases under stressed conditions (Dong et al., 2017; Eissa et al., 2015). The present study was carried out to isolate and identify the causative agent of fish diseases and mortalities outbreak in tilapia fish in farming culture in Egypt.

Naturally infected Nile tilapia fish showing hemorrhagic skin, body depigmentation, frayed fins and tail, corneal opacity, exophthalmia, body ulceration, detachment of scales, hemorrhages over the fish body especially 
Table (1): The morphological and biochemical characters of isolated Gram-negative bacteria (Aeromonas spp., Pseudomonas spp., and Vibrio spp.) from the examined Nile tilapia fish.

\begin{tabular}{|c|c|c|c|c|c|c|c|c|c|c|c|}
\hline \multirow[t]{2}{*}{ Identification Test } & \multicolumn{5}{|c|}{ Aeromonas spp. } & \multicolumn{3}{|c|}{ Pseudomonas spp. } & \multicolumn{3}{|c|}{ Vibrio spp. } \\
\hline & 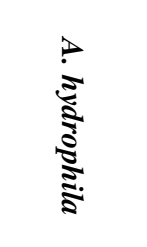 & 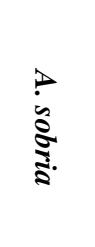 & 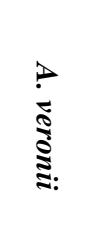 & 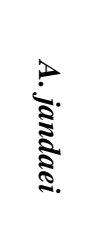 & ?. & 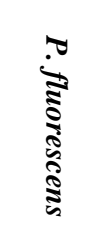 & 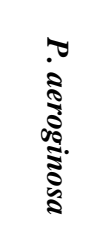 & 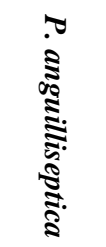 & 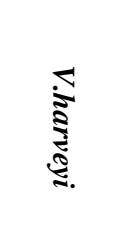 & 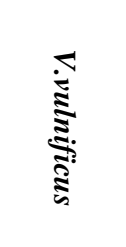 & 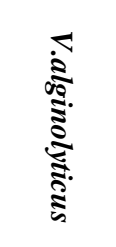 \\
\hline Shape & Short rod & $\begin{array}{c}\text { Short } \\
\text { rod }\end{array}$ & $\begin{array}{c}\text { Short } \\
\text { rod }\end{array}$ & $\begin{array}{c}\text { Short } \\
\text { rod }\end{array}$ & Short rod & $\begin{array}{c}\text { Long } \\
\text { curved } \\
\text { rods }\end{array}$ & $\begin{array}{l}\text { Long } \\
\text { curved } \\
\text { rods }\end{array}$ & $\begin{array}{l}\text { Long } \\
\text { curved } \\
\text { rods }\end{array}$ & $\begin{array}{l}\text { curved } \\
\text { rods }\end{array}$ & $\begin{array}{l}\text { curved } \\
\text { rods }\end{array}$ & $\begin{array}{l}\text { curved } \\
\text { rods }\end{array}$ \\
\hline Motility & + & + & + & + & + & + & + & + & + & + & + \\
\hline Cytochrom oxidase & + & + & + & + & + & + & + & + & + & + & + \\
\hline $\mathrm{O} / \mathrm{F}$ & $\mathrm{F}$ & $\mathrm{F}$ & $\mathrm{F}$ & $\mathrm{F}$ & $\mathrm{F}$ & $\mathrm{O}$ & $\mathrm{O}$ & - & $\mathrm{F}$ & $\mathrm{F}$ & $\mathrm{F}$ \\
\hline Growth on TCBS & & & & & & & & & $\begin{array}{l}\text { Yellow } \\
\text { colonies }\end{array}$ & $\begin{array}{l}\text { Green } \\
\text { colonies }\end{array}$ & $\begin{array}{c}\text { Yellow } \\
\text { colonies }\end{array}$ \\
\hline Growth at $5^{\circ} \mathrm{C}$ & + & + & - & - & + & + & - & + & & & \\
\hline Growth on $0 \% \mathrm{NaCl}$ & + & + & + & + & + & + & + & + & + & - & - \\
\hline $3.5 \% \mathrm{NaCl}$ & + & - & + & + & + & + & + & + & + & + & + \\
\hline $6 \% \mathrm{NaCl}$ & - & - & - & - & - & + & + & - & + & + & + \\
\hline $8 \% \mathrm{NaCl}$ & & & & & & & & & - & + & + \\
\hline $10 \% \mathrm{NaCl}$ & & & & & & & & & - & - & + \\
\hline Catalase & + & + & + & + & + & + & + & + & + & + & + \\
\hline $\mathrm{H}_{2} \mathrm{~S}$ (TSI) & + & - & - & - & - & - & - & - & - & - & + \\
\hline
\end{tabular}


Table (1): Continued.

\begin{tabular}{|c|c|c|c|c|c|c|c|c|c|c|c|}
\hline \multirow[t]{2}{*}{ Identification Test } & \multicolumn{5}{|c|}{ Aeromonas spp. } & \multicolumn{3}{|c|}{ Pseudomonas spp. } & \multicolumn{3}{|c|}{ Vibrio spp. } \\
\hline & 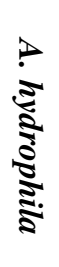 & 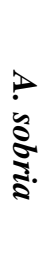 & 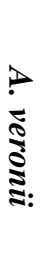 & 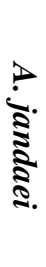 & 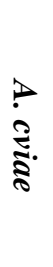 & 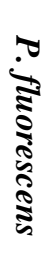 & 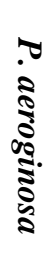 & 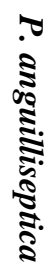 & 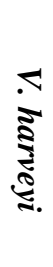 & 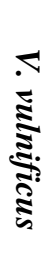 & 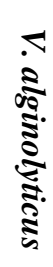 \\
\hline Indol & + & + & + & + & + & - & - & - & + & - & + \\
\hline Urease & - & - & - & - & - & + & + & - & & & \\
\hline Starch hydrolysis & - & + & $\mathrm{v}$ & - & + & - & - & - & & & \\
\hline Methyl red & + & $\mathrm{v}$ & $\mathrm{v}$ & + & - & $\mathrm{v}$ & - & + & + & + & + \\
\hline Vogaus proskauer & + & - & + & + & - & - & - & - & - & - & + \\
\hline Citrate & + & + & + & + & + & + & + & + & + & + & - \\
\hline Gelatin liquefaction & + & $\mathrm{v}$ & + & - & + & + & + & + & - & + & $\mathrm{v}$ \\
\hline Ornithen decarboxylase & - & - & + & + & - & - & & & - & + & + \\
\hline Lysin decarboxylase & + & + & + & + & - & - & - & - & & - & + \\
\hline Arginie dehydrogenase & + & - & $\mathrm{v}$ & - & + & + & + & + & - & - & + \\
\hline \multicolumn{12}{|l|}{ Acid production from } \\
\hline Arabinose & + & - & - & - & + & + & - & - & - & - & - \\
\hline Salicin & $\mathrm{v}$ & - & + & - & + & - & & - & - & + & - \\
\hline Sucrose & + & + & + & - & + & + & - & - & + & - & + \\
\hline Inositol & - & - & - & - & - & - & - & - & + & - & - \\
\hline Glucose & + & + & + & + & - & + & - & - & + & + & + \\
\hline Maltose & + & + & + & + & + & - & + & + & & & \\
\hline Mannitol & + & + & + & + & + & + & - & - & + & - & + \\
\hline glycerol & + & + & + & + & $\mathrm{v}$ & & & & & & \\
\hline lactose & & & & & & & & & - & $\mathrm{v}$ & - \\
\hline
\end{tabular}


Table (2): The morphological and biochemical characters of isolated Gram-positive bacteria from the examined Nile tilapia fish.

\begin{tabular}{|c|c|c|}
\hline Identification Test & Staphylococcus sp. & Streptococcus sp. \\
\hline Gram-stain & +ve & $+\mathrm{ve}$ \\
\hline Shape & Cocci & Cocci \\
\hline Motility & - & - \\
\hline Cytochrom oxidase & - & - \\
\hline $\mathrm{O} / \mathrm{F}$ & $\mathrm{O}$ & $\mathrm{F}$ \\
\hline Growth at $5^{\circ} \mathrm{C}$ & + & - \\
\hline Growth on $0.0 \% \mathrm{NaCl}$ & + & + \\
\hline $3.5 \% \mathrm{NaCl}$ & + & + \\
\hline $6 \% \mathrm{NaCl}$ & + & + \\
\hline $8 \% \mathrm{NaCl}$ & + & - \\
\hline Catalase & + & - \\
\hline $\mathrm{H}_{2} \mathrm{~S}(\mathrm{TSI})$ & - & - \\
\hline indole & - & - \\
\hline Urease & + & - \\
\hline Starch hydrolysis & - & + \\
\hline Methyl red & + & - \\
\hline Vogaus proskauer & + & - \\
\hline Citrate & + & - \\
\hline Gelatin liquefaction & + & - \\
\hline Heamolysis & $\beta$ & $\beta$ or $\gamma$ \\
\hline Alkaline phosphotase & + & + \\
\hline Ornithen decarboxylase & - & + \\
\hline Lysin decarboxylase & - & - \\
\hline Arginie dehydrogenase & + & + \\
\hline \multicolumn{3}{|l|}{ Acid production from: } \\
\hline Arabinose & - & $\mathrm{v}$ \\
\hline Salicin & - & $\mathrm{v}$ \\
\hline Sucrose & + & + \\
\hline Inositol & - & - \\
\hline Glucose & + & + \\
\hline Maltose & + & + \\
\hline Mannitol & + & $\mathrm{v}$ \\
\hline Lactose & + & $\mathrm{V}$ \\
\hline Fructose & + & + \\
\hline
\end{tabular}




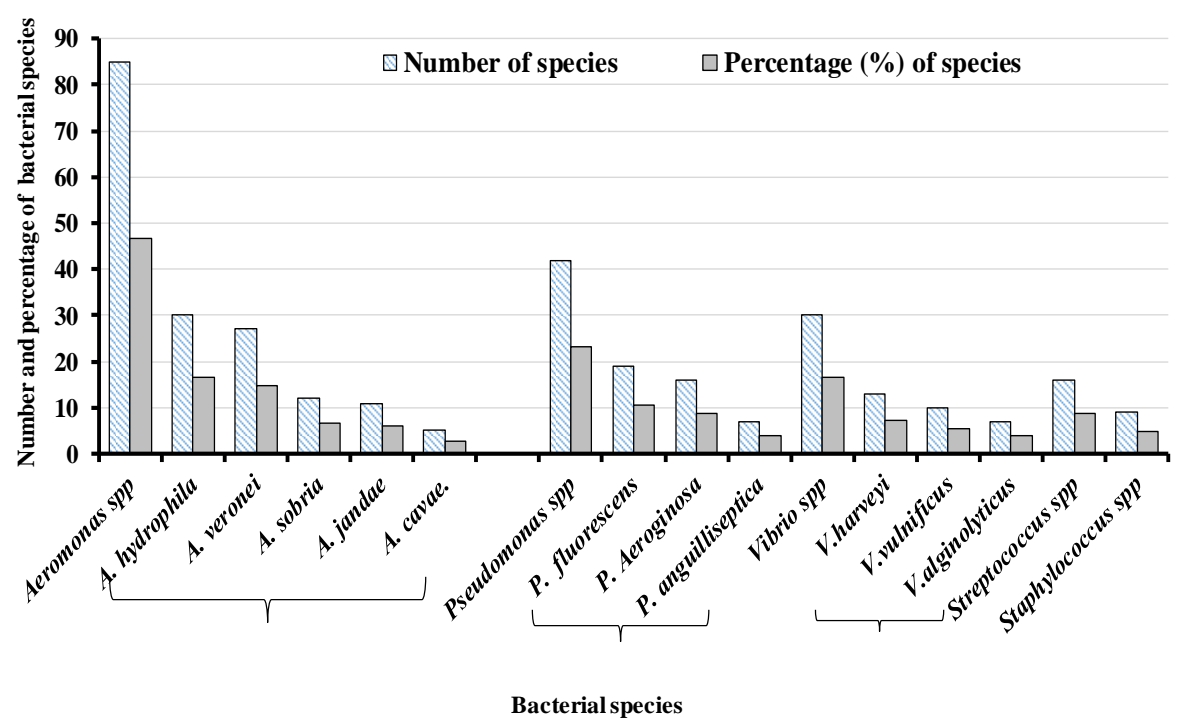

Figure (3): Prevalence of isolated bacteria in the examined fish

at fins and tails, erythema around the mouth and swelling of the abdomen. Postmortem examination showing congection of the internal organs and presence of ascetic fluid. These abnormalities are similar to that were reported by Pretto-Giordano et al., (2010) and El-Son, (2016) who observed signs such as anorexia, exophthalmia, skin alterations, an extension of the visceral cavity, corneal opacity, bleeding, and abdominal inflammation, splenomegaly and hepatomegaly. Soto, (2009) reported that the bacteria are the main cause of splenomegaly, epithelial hyperplasia in gills and necrosis in internal organs mainly in kidney, liver, spleen, musculature, heart, and brain.

In this study, the bacteriological examination of infected fishes showed the isolation of three Gram-negative bacteria at $86.26 \%$ of the total isolated strains with the predominance of Aeromonas spp. at $46.70 \%$. Besides, two Gram-positive bacteria at $13.74 \%$ were isolated with the dominance of Streptococcus spp. at $8.79 \%$. These results are following some researches who reported that Aeromonas spp., Pseudomonas spp., Vibrio spp., Streptococcus spp., Staphylococcus spp., Micrococcus spp. and Enterobacteriaceae were responsible for the fish fatal outbreak (Daskalov, 2006: Najiah et al 2012). El-Gamal et al., (2018) also reported that the bacteria isolated from naturally infected $O$. niloticus from different fish farms at Kafr El-Sheikh Governorate, Egypt, were Aeromonas spp. by 26\%, 
Table (3): Incidence of isolated bacterial from the examined organs of Nile tilapia fish

\begin{tabular}{|c|c|c|c|c|c|c|c|c|c|c|c|c|}
\hline & \multicolumn{2}{|c|}{ Aeromonas sp. } & \multicolumn{2}{|c|}{ Pseudomonas sp. } & \multicolumn{2}{|c|}{ Vibrio sp. } & \multicolumn{2}{|c|}{ Streptococcus sp. } & \multicolumn{2}{|c|}{ Staphylococcus sp. } & \multicolumn{2}{|c|}{ Total } \\
\hline Organ & $\begin{array}{l}\text { No. of } \\
\text { isolates }\end{array}$ & $\%$ & $\begin{array}{l}\text { No. of } \\
\text { isolates }\end{array}$ & $\%$ & $\begin{array}{l}\text { No. of } \\
\text { isolates }\end{array}$ & $\%$ & $\begin{array}{l}\text { No. of } \\
\text { isolates }\end{array}$ & $\%$ & $\begin{array}{l}\text { No. of } \\
\text { isolates }\end{array}$ & $\%$ & $\begin{array}{l}\text { No. of } \\
\text { isolates }\end{array}$ & $\%$ \\
\hline Skin ulcer & 3 & 3.53 & 1 & 2.38 & 2 & 6.67 & 1 & 6.25 & 0 & 0.00 & 7 & 3.84 \\
\hline Tail & 4 & 4.70 & 3 & 7.14 & 3 & 10.0 & 2 & 12.5 & 1 & 11.1 & 13 & 7.14 \\
\hline Liver & 35 & 41.1 & 21 & 50.0 & 7 & 23.3 & 5 & 31.2 & 0 & 0.00 & 68 & 37.3 \\
\hline Kidney & 23 & 27.0 & 11 & 26.1 & 6 & 20.0 & 2 & 12.5 & 5 & 55.5 & 47 & 25.8 \\
\hline Spleen & 11 & 12.9 & 3 & 7.14 & 8 & 26.6 & 4 & 25.0 & 2 & 22.2 & 28 & 15.3 \\
\hline Gills & 9 & 10.5 & 3 & 7.14 & 4 & 13.3 & 2 & 12.5 & 1 & 11.1 & 19 & 10.4 \\
\hline Total & 85 & 46.7 & 42 & 23.08 & 30 & 16.4 & 16 & 8.79 & 9 & 4.95 & 182 & 100 \\
\hline
\end{tabular}


Table (4): Antibiogram sensitivity test.

\begin{tabular}{|c|c|c|c|c|c|c|c|c|c|}
\hline Antibiotic tested & & NOR & CIP & $\mathbf{G N}$ & Amx & Ak & RF & $\mathbf{E}$ & $\mathbf{A M}$ \\
\hline Concentration & & $10 \mathrm{mg}$ & $5 \mathrm{mg}$ & $10 \mu \mathrm{g}$ & $25 \mu \mathrm{g}$ & $30 \mathrm{mg}$ & $30 \mu \mathrm{g}$ & $15 \mu \mathrm{g}$ & $10 \mu \mathrm{g}$ \\
\hline \multirow{3}{*}{$\begin{array}{l}\text { Zone diameter } \\
\text { interpretation } \\
\text { Standards (mm) }\end{array}$} & $\mathbf{R}$ & $\geq 12$ & $\geq 15$ & 12 & $\geq 11$ & $\geq 14$ & $\geq 16$ & $\geq 13$ & $\geq 13$ \\
\hline & $\mathbf{I}$ & 13-16 & $16-20$ & 13-14 & $12-13$ & $15-16$ & 17-19 & 14-22 & 14-16 \\
\hline & $\mathbf{S}$ & $\leq 17$ & $\leq 21$ & 15 & $\leq 14$ & $\leq 17$ & $\leq \mathbf{2 0}$ & $\leq \mathbf{2 3}$ & $\leq 17$ \\
\hline A. hydrophila & & $18(\mathrm{~S})$ & $25(\mathrm{~S})$ & $17(\mathrm{~S})$ & $10(\mathrm{R})$ & $19(\mathrm{~S})$ & $13(\mathrm{R})$ & $0(\mathrm{R})$ & $0(\mathrm{R})$ \\
\hline A. veronei & & $24(\mathrm{~S})$ & $27(\mathrm{~S})$ & $19(\mathrm{~S})$ & $0(\mathrm{R})$ & 13(R) & $17(\mathrm{I})$ & $0(\mathrm{R})$ & $0(\mathrm{R})$ \\
\hline A. sobria & & $32(\mathrm{~S})$ & $29(\mathrm{~S})$ & $14(\mathrm{I})$ & $6(\mathrm{R})$ & $18(\mathrm{~S})$ & $16(\mathrm{R})$ & $11(\mathrm{R})$ & $0(\mathrm{R})$ \\
\hline A. jandae & & $24(\mathrm{~S})$ & $22(\mathrm{~S})$ & $18(\mathrm{~S})$ & $0(\mathrm{R})$ & $26(S)$ & $18(\mathrm{I})$ & $13(\mathrm{R})$ & $0(\mathrm{R})$ \\
\hline A. caviae & & $27(\mathrm{~S})$ & $28(\mathrm{~S})$ & $15(\mathrm{~S})$ & $10(\mathrm{R})$ & $21(\mathrm{~S})$ & $14(\mathrm{R})$ & $0(\mathrm{R})$ & $0(\mathrm{R})$ \\
\hline P. fluorescens & & $18(\mathrm{~S})$ & $22(\mathrm{~S})$ & $13(\mathrm{I})$ & $7(\mathrm{R})$ & $23(\mathrm{~S})$ & $9(\mathrm{R})$ & $7(\mathrm{R})$ & $0(\mathrm{R})$ \\
\hline P. Aeroginosa & & $20(S)$ & $15(\mathrm{R})$ & $16(S)$ & $8(\mathrm{R})$ & $21(\mathrm{~S})$ & $17(\mathrm{I})$ & $23(\mathrm{~S})$ & $0(\mathrm{R})$ \\
\hline P. anguilliseptica & & $19(\mathrm{~S})$ & $21(\mathrm{~S})$ & $19(\mathrm{~S})$ & $0(\mathrm{R})$ & $0(\mathrm{R})$ & $15(\mathrm{R})$ & $19(\mathrm{I})$ & $0(\mathrm{R})$ \\
\hline V. harveyi & & $24(\mathrm{~S})$ & $28(\mathrm{~S})$ & $16(S)$ & $0(\mathrm{R})$ & $21(\mathrm{~S})$ & $20(\mathrm{~S})$ & $10(\mathrm{R})$ & $7(\mathrm{R})$ \\
\hline V. vulnificus & & $17(\mathrm{~S})$ & $26(S)$ & $20(\mathrm{~S})$ & $9(\mathrm{R})$ & $19(\mathrm{~S})$ & $16(\mathrm{R})$ & $9(\mathrm{R})$ & $0(\mathrm{R})$ \\
\hline V. alginolyticus & & $20(\mathrm{~S})$ & $22(\mathrm{~S})$ & $17(\mathrm{~S})$ & $0(\mathrm{R})$ & $17(S)$ & $23(\mathrm{~S})$ & $0(\mathrm{R})$ & $0(\mathrm{R})$ \\
\hline Streptococcus spp. & & $21(\mathrm{~S})$ & $13(\mathrm{R})$ & $6(\mathrm{R})$ & $14(\mathrm{~S})$ & $16(\mathrm{I})$ & $18(\mathrm{I})$ & $9(\mathrm{R})$ & $0(\mathrm{R})$ \\
\hline Staphylococcus spp. & & $18(\mathrm{~S})$ & $16(\mathrm{I})$ & $17(S)$ & $12(\mathrm{I})$ & $18(S)$ & $21(\mathrm{~S})$ & $7(\mathrm{R})$ & $7(\mathrm{R})$ \\
\hline
\end{tabular}

NOR= Norfloxacin, $\mathrm{CIP}=$ Ciprofloxacin, $\mathrm{GN}=$ Gentamycin, $\mathrm{AMX}=$ Amoxicillin, AK= Amikacin, RF= Rifamycin, E= Erythromycin , AM= Ampicillin, $\mathrm{R}=$ Resistant, $\mathrm{S}=$ Sensitive, $\mathrm{I}=$ Intermediate 
Pseudomonas spp. by $23.3 \%$, Staphylococcus aureus by $7.3 \%$ and mixed infections were $36.6 \%$ and they are responsible for ulcerative syndrome. This indicated that the outbreak is mainly attributed to the same genera as previously. Therefore, controlling these strains is pending necessary to prevent further loss of fish culture. A previous study in Indonesia by Hardi., et al. (2018) reported that the Gram-negative bacteria are the most predominant bacteria found in cultured tilapia. They have isolated seven bacterial genera from tilapia and catfish (Streptococcus sp., Staphylococcus sp., Pseudomonas sp., Enterobacter sp., Aeromonas sp., Neisseria sp. and Listeria sp.).

In the present study, Aeromonas spp. were the predominant pathogenic bacteria followed by genus Pseudomonas spp. Austin and Austin, (2012) reported that Aeromonas spp. infect fishes and were responsible for the Motile Aeromonas Septicemia (MAS) disease in fish or epizootic ulcerative syndrome (EUS). Also, some researchers reported that Pseudomonas spp. (P. fluorescens, P. aeruginosa, P. putida, and P. angulliseptica) were the causative agents of Pseudomonas septicemia in various species of fish (Eissa et al., 2010; EL-Nagar, 2010)

In this study, Aeromonas hydrophila had the highest prevalence rate of isolated bacteria. This is agreed with Hassan et al., (2017), who reported that the Aeromonas hydrophila was the most predominant Aeromonas spp. found in tilapia aquaculture. Van Hai, (2015) also reported that Aeromonas hydrophila was the bacterial pathogen in aquaculture that results in huge economic losses and causing a disease known as hemorrhagic septicemia or motile septicemia.

The present study indicated that the incidence of isolated bacteria from examined organs of fish was higher in the liver followed by kidney and spleen. Mahmoud et al., (2016) also reported that bacterial isolates distribution in different organs and tissues of the examined fishes were presented mainly in the liver, kidney, and spleen. Ezzat et al., (2018) have reported that the kidney and the liver were the most predominant organs for isolation of bacteria at a rate $(37.4 \%)$ and $(36.3 \%)$, respectively followed by spleen (15.9\%) and gills (9.85\%). Eissa et al., (2016) reported that Aeromonas sobria isolates were isolated with the high prevalence from kidneys $25.3 \%$, liver $23.0 \%$, spleen $19.8 \%$ and intestines $15.0 \%$ and the lowest prevalence was recorded from gills and skin lesions at rates of $10.3 \%$ and $6.35 \%$ respectively. Eissa et al., (2010) also reported that the prevalence of Pseudomonas sp. in organs of Nile tilapia was mainly from the liver (35\%) and kidney (30\%) followed by spleen (21.2\%) and gills 
(13.7\%). EL-Sayed et al., (2019) also reported that the prevalence of $V$. alginolyticus was high in liver at rate $38.5 \%$ followed by kidney $29.2 \%$, spleen $23 \%$ and heart $9.2 \%$ from naturally infected $O$. niloticus. The occurrence of Staphylococcus $s p$. in this study was mainly from kidney and spleen, but not isolated from liver and skin ulcer. While Osman et al., (2017) reported that Streptococcus spp. occurred mainly in 8/80 (10\%) in liver samples followed by 4/80 (5\%) in spleen samples, 3/80 (3.8\%) in kidney samples and 1/80 (1.3\%) in brain and ascitic fluid samples in Nile tilapia. This result was different from result recorded by Ali, (2014) who reported that Staphylococcus spp. was most commonly detected in skin $(35.5 \%, 36.8 \%)$, livers $(25.8 \%, 25 \%)$, intestines $(21 \%, 17.60 \%)$, muscles $(17.7 \%, 20.6 \%)$ of Cyprinus carpio and Silurus glanis, respectively.

The results of sensitivity tests in the current study showed that all or most tested strains were sensitive to norfloxacin or ciprofloxacin. Therefore, these antibiotics can be used for the treatment of infected fishes against these bacteria. On the other hand, high levels of resistance were expressed by all isolates to ampicillin, amoxicillin, and erythromycin indicating that these antibiotics are inappropriate to treat fishes infected with pathogenic bacteria. Similarly, El-Barbary and Hal, (2016) reported that ciprofloxacin, norfloxacin, and gentamycin could be used to treat fish infected with A. hydrophila, P. fluorescens, P. putida, and Klebsiella oxytoca.

Efforts are needed to control the disease from occurring rather than treating the disease which is most of the time risky and expensive. The wide and frequent application of antibiotics in aquaculture not only results in antibiotics resistance in aquatic bacteria but also residual antibiotics in the environmental and aquatic products (Lalumera et al., 2004). Such residual antibiotics may cause allergic reactions, toxicity, and alteration of normal microflora of consumers and results in antibiotics resistance in bacterial pathogens (Cabello, 2006). Therefore, searching for a safe alternative to the use of antibiotics is of great importance (Kesarcodi-Watson et al., 2008). Several medicinal plants have attracted great attention as a safe alternative to synthetic chemotherapeutics (Mehrim and Salem, 2013). They are advantageous as natural substances that have no effect on the health of fish, humans, and environment, of low cost, and would be minimizing the side effects compared to synthetic chemotherapeutics (Gabor et al., 2010). Thus, further studies based on the enhancement haemato-biochemical and immune parameters of Nile tilapia against challenge with pathogenic bacteria are under investigation. 


\section{CONCLUSION}

The present study concluded that the Gram-negative bacteria were the main cause of Nile tilapia diseases and mortality in farmed fish. A. hydrophila exhibited the highest prevalence in naturally infected Nile tilapia fish. The bacterial infection occurs mainly in the liver and kidney of infected fish. Norfloxacin was drug of choice to treat fish infected with bacterial pathogens. Further investigations on the safe effective supplements, alternatives to antibiotics, for improving growth performance and immunity against bacterial pathogens should be recommended.

\section{ACKNOWLEDGEMENTS}

We would like to thank Prof. Ahmed Darwesh El-Gamal, Professor of phycology, Faculty of Science, Al-Azhar University and Dr. Somayah M.M. Awad, Senior Researcher at Department of Fish Health and Management, Central Laboratory for Aquaculture Research, Abbassa, Abo-Hammad, Sharqia, Egypt for their great support and contributions through this work.

\section{CONFLICT OF INTEREST STATEMENT}

The authors declare that there are no conflicts of interest.

\section{DATA AVAILABILITY}

The data used to support the findings of this study are available from the corresponding author upon request.

\section{FUNDING}

This work was not supported by any funding.

\section{REFERENCES}

Abd El-Kader M., and Balabel, M. T. M. (2017). Isolation and Molecular Characterization of Some Bacteria Implicated in the Seasonal Summer Mortalities of Farm-raised Oreochromis niloticus at Kafr El-Sheikh and Dakahlia Governorates. A.J.V.S. 53, (2), 107-113.

Ali, H. H. (2014). Isolation and identification of Staphylococcus bacteria from fish of fresh water and its antibiotics sensitivity in Mosul city. Bas.J.Vet.Res.Vol.1, No.1.32-42.

Austin, B., and Austin, D. (2012). Aeromonadaceae Representative (Aeromonas salmonicida). Bacterial Fish Pathogens. (Netherlands: Springer), 147-228. 
Briede, I. (2010). The prevalent bacterial fish diseases in fish hatcheries of Lativia. Environ. Exp. Biol. 8, 103-106.

Cabello, F. C. (2006). Heavy use of prophylactic antibiotics in aquaculture: growing problem for human and anemia health and for the environment. Environ. Microboil. 8, 1137-1144.

Daskalov, H. (2006). The importance of Aeromonas hydrophila in food safety. Food Control. 17, 474-483.

Dong, H. T., Techatanakitarnan, C., Jindakittikul, P., Thaiprayoon, A., Taengphu, S., Charoensapsri, W., and Senapin, S. (2017). Aeromonas jandaei and Aeromonas veronii caused disease and mortality in Nile tilapia, Oreochromis niloticus (L.). Journal of Fish Diseases, 40(10), 1395-1403.

Eissa I. A. M., El-lamei, M., Isamil, T., Youssef, F.and Mansour, S. (2016). Advanced Studies for Diagnosis of Aeromonas Septicemia in Oreochromis niloticus. SCVMJ, XXI (1), 221-233.

Eissa, I. A. M., Maather, E. I., Mona, S., Desuky, E., Mona, Z., and Bakry, M. (2015). Aeromonas veronii biovar sobria a causative agent of mass mortalities in cultured Nile Tilapia in El-Sharkia governorate, Egypt. Life Science Journal. 12, 90-97.

Eissa, N. M. E., Abou El-Ghiet, E. N., Shaheen, A. and Abbass, A. (2010). Characterization of Pseudomonas Species Isolated from Tilapia "Oreochromis niloticus" in Qaroun and Wadi-El-Rayan Lakes, Egypt. Glob. Vet., 5: 116-121.

El-Barbary, M. I. and Hal, A. M. (2016). Isolation and molecular characterization of some bacterial pathogens in El-Serw fish farm, Egypt. Egypt. J. Aquat. Biol. \& Fish. 20, (4), 115-127.

El-Gamal, A. m., El-Gohary, M. S. and Gaafar, A. Y. (2018). Detection and Molecular Characterization of Some Bacteria Causing Skin Ulceration in Cultured Nile Tilapia (Oreochromis niloticus) in Kafr ElSheikh Governorate. Int. J. Zool. Res., 14 (1): 14-20.

El-Nagar, R. M. A. (2010). Bacteriological studies on Pseudomonas microorganisms in cultured fish. MSc. thesis, Fac. Vet. Med., Zag. University.

EL-Sayed, M. E., Al gammal, A. M., Abouel-Atta, M. E. and Mabrok, A. M. (2019). Pathogenicity, genetic typing, and antibiotic sensitivity of Vibrio alginolyticus isolated from Oreochromis niloticus and Tilapia zillii. Revue Méd. Vét. 170, 4-6, 80-86. 
El-Son, M. A. (2016): Effect of some environmental parameters on spreading of bacterial fish diseases. PhD Thesis, Fac. Vet. Med, Mansoura University, Egypt.

Ezzat, E. M., Essawy, M., Shabana, I. I., Abou El-Atta, M. and Banna, N. (2018). Studies on Bacterial Pathogens in Some Marine Fishes in ELMansoura, Egypt. American Journal of Agricultural and Biological Sciences.13 (1): 9.15 DOI: 10.3844/ajabssp.2018.9.15

FAO, (2019). The state of world fisheries and aquaculture. FAO Fisheries department, fisheries information, data and statistics unit, Rome.

Gabor, E. F., Sara, A. and Barbu, A. (2010). The effects of some phytoadditives combination om growth, health and meat quality on different species of fish. Scientific paper: Animal Science and Biotechnologies. 43(1),61-65.

GAFRD. (2017). Egyptian Fish statistics year book, 2015 (27 ed.). Cairo, Egypt, General Authority for Fish Resources Development.

Hardi, E. H., Nugroho, R. A., Saptiani, G., Sarinah, R., Agriandini, M. and Mawardi, M. (2018). Identification of potentially pathogenic bacteria from tilapia (Oreochromis niloticus) and channel catfish (Clarias batrachus) culture in Samarinda, East Kalimantan, Indonesia. BIODIVERSITAS 19 (2), 480-488.

Hassan, M. A., Noureldin, E. A., Mahmoud, M. A., and Fita, N. A. (2017). Molecular identification and epizootiology of Aeromonas veronii infection among farmed Oreochromis niloticus in Eastern Province, KSA. Egyptian Journal of Aquatic Research. 43 (2), 161-167.

Hassan, M. N., EL-Ashram, A. M. M., and Mahmoud, W. G. (2011). Detection and characterization of virulence factors among Aeromonas species isolated from diseased fish. Zag. Vet. J. 39, (2),113-121.

Kesarcodi-Watson, A., Kaspar, H., Lategan, M. J. and Gibson, L. (2008). Probiotics in aquaculture: the end mechanisms of action and screening processes. Aquaculture. 274, 1-14.

Lalumera, G. M., Calamari, D., Galli, P., Castiglioni, S., Crosa, G. and Fanelli, R. (2004). Preliminary investigation on the environmental occurrence and effects of antibiotics used in aquaculture in Italy. Chemosphere. 54, 661-668.

Mahmoud, M. A., Abdelsalam, M., Mahdy, O. A., El Miniawy, H. M. F., and Z.A.M. Osaman, A. H., Mohamed, H. M. H., Khattab, A. M. and Ewiss, M. A. (2016). Infectious bacterial pathogens, parasites and 
pathological correlations of sewage pollution as an important threat to farmed fishes in Egypt. Environ.Pollut. 219, 939-948.

Mapenzi, L. L. and Mmochi, A. J. (2016). Role of salinity on growth performance of Oreochromis niloticus $q$ and Oreochromis urolepisurolepis $\widehat{\jmath}$ Hybrids. J. Aquac. Res. Development.7, 431.

Mehrim, A. I. and Salem, M. F. (2013). Medicinal herbs against aflatoxicosis in Nile tilapia (Orechromis niloticus): Clinical signs, postmortem lesions and liver histopathological changes. Egyptian Journal for Aquaculture. 3, 13-15.

Najiah, M., Aqilah, N., Lee K. L., Khairulbariyyah, Z., Mithun, S., Jalal, K. C. A., Shaharom-Harrison, F. and Nadiah, M. (2012). Massive Mortality Associated with Streptococcus agalactiae Infection in Cage-cultured Red hybrid Tilapia Oreochromis niloticus in Como River, Kenyir Lake, Malaysia. J. Biol. Sci. 12, (8), 438-442.

National Committee of Clinical Laboratory Standards (NCCLS) (2003). "Performance standards for antimicrobial disk and dilution susceptibility tests for bacteria isolated from animals". Approved standard, (NCCLS document M31-A2, Wayne, PA).

Noga, E. J. (2010). Fish disease: Diagnosis and treatment. 2nd ed.WileyBlackwell publication, USA.

Osman, K. M., Al-Maary, K. S., Mubarak, A. S., Dawoud, T. M., Moussa, I. M. I, Ibrahim, M. D. S., Hessain, A. M. Orabi, A. and Fawzy, N. M. (2017). Characterization and susceptibility of streptococci and enterococci isolated from Nile tilapia (Oreochromis niloticus) showing septicaemia in aquaculture and wild sites in Egypt. BMC Veterinary Research.13:357.

Plumb, J. A., and Hanson, L. A. (2010): Health maintenance and principal microbial diseases of cultured fishes. Wiley-Blackwell, Hoboken, NJ.

Pretto-Giordano, L. G., Eckehard-Müller, E., Freitas, J. C. D., and da Silva, V. G. (2010): Evaluation on the Pathogenesis of Streptococcus agalactiae in Nile Tilapia (Oreochromis niloticus). Braz Arch Biol Techn. 53, 87-92.

Pridgeon, J. W., Klesius, P. H., Mu, X. and Song, L. (2011). Anin vitro screening method to evaluate chemicals as potential chemotherapeutants to control Aeromonas hydrophila infection in channel catfish. J Appl Microbio.1111, 114-124. 
Quinn, P. T., Markey, B. K., Carter, M. E., Donnelly, W. J., and Leonard, F. C. (2002): Veterinary Microbiology and Microbial disease. First Published Blackwell Science Company, Lowa, State University Press.

Shaheen, A., Seisay, M., and Nouala, S. (2013): An industry assessment of Tilapia farming in Egypt. African Union, International Bureau for Animal Resources (AU-IBAR).

Soto, S. A. (2009): Water quality and bacteria present in cultivated tilapia. Foundation produces Tilapia A.C pp: 7-19.

Van Hai, N. (2015): Research findings from the use of probiotics in tilapia aquaculture: a review. Fish Shellfish Immunol. 45, 592-597.

Zahran, E., Manning, B., Seo, J. K., Noga, E. J. (2016). The effect of ochratoxin A on antimicrobial polypeptide expression and resistance to water mold infection in channel catfish (Ictaluru spunctatus). Fish Shellfish Immunol. 57, 60-67. 


\section{انتشار ومدى قابلية المضادات الحيوية للبكتيريا الممرضة والمتسبية في نفوق اسماك

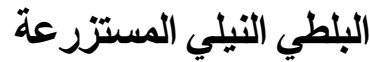

سعد الدين حسن1 ، محمد على عبد الرحمن1 ، السيد منصور22 ، وليد منير1

1ققم النبات و الميكروبيولوجى، كلية العلوم بنين ، جامعة الأزهر، القاهرة

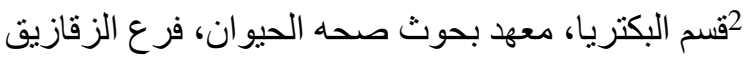

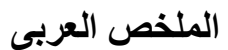

تهدف هذه الدراسة الي عزل وتوصيف البكتريا الممرضة المسببة للنفوق في سمك المك البلطي النيلي، تم تجميع عدد . . 1 عينه من سمكة البلطي النيلي تحمل علامات مرضية تم فحصها اكلينيكيا وتثريحيا وتم عمل الفحوصات البكتيرية وكذلك تم عمل اختبار الحساسية للمضادات

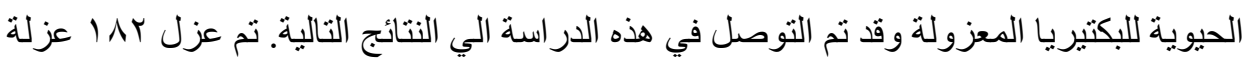

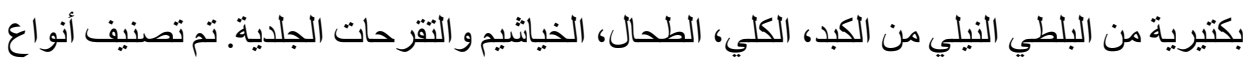

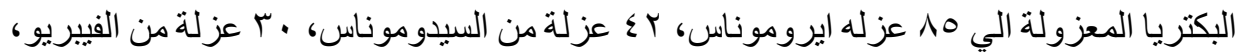
17 عزلة من ستربتوكوكس و9 عزلة من ستافيلوكوكس. وتم تصنيف العزلات من سلالة

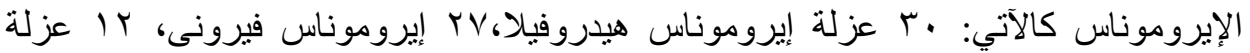
إيروموناس سوبريا، 1 ا عزلة إيروموناس جاندي وه عزلة إيروموناس كافى:وتم تصنيف العز لات من سلالة السيدوموناس كلآتى: 19 عزلة من السيدوموناس فلوريسينس , 17 عزلة من السيدوموناس ايرويجنوز او V عزلة من السيدوموناس انجويليسبتيكا وتصينف العز لات من سلالة الفيبريو كالآتي: rا عزلة فييريوهارفي ، · ل عزلة فييريو فولنيفكس وV عزلة فيبريو

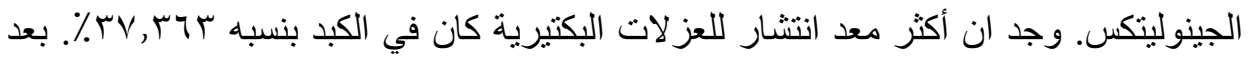
عمل اختبار الحساسية للمضادات الحيويه وجد ان العزلات حساسة للنورفلوكساسين، السييروفلوكساسين، الجنتاميسين والأميكاسين بينما أظهرت العزلات مقاومة لريفاميسين، الاريثرو مايسين، اموكسيسيلن و امبسيلين. 